\title{
Demarcating mobile phone interface design guidelines to expedite selection
}

\author{
Karen Renaud $^{\mathrm{a}, \mathrm{b}}$, Judy van Biljon ${ }^{\mathrm{b}}$ \\ ${ }^{\text {a }}$ Abertay University, Dundee, Scotland \\ ${ }^{b}$ University of South Africa, Pretoria, South Africa
}

\begin{abstract}
Guidelines are recommended as a tool for informing user interface design. Despite a proliferation of guidelines in the research literature, there is little evidence of their use in industry, nor their influence in academic literature. In this paper, we explore the research literature related to mobile phone design guidelines to find out why this should be so. We commenced by carrying out a scoping literature review of the mobile phone design guideline literature to gain insight into the maturity of the field. The question we wanted to explore was: "Are researchers building on each others' guidelines, or is the research field still in the foundational stage?" We discovered a poorly structured field, with many researchers proposing new guidelines, but little incremental refinement of extant guidelines. It also became clear that the current reporting of guidelines did not explicitly communicate their multi-dimensionality or deployment context. This leaves designers without a clear way of discriminating between guidelines, and could contribute to the lack of deployment we observed. We conducted a thematic analysis of papers identified by means of a systematic literature review to identify a set of dimensions of mobile phone interface design guidelines. The final dimensions provide a mechanism for differentiating guidelines and expediting choice.
\end{abstract}

Keywords: guidelines, mobile phone design, interaction design

Categories: • Human-centred computing $\sim$ User interface design

Email:

Karen Renaud k.renaud@abertay.ac.uk (CORRESPONDING),

Judy van Biljon vbiljja@unisa.ac.za

\author{
Article history: \\ Received: 26 Nov 2016 \\ Accepted: 21 Nov 2017 \\ Available online: 8 Dec 2017
}

\section{INTRODUCTION}

Guidelines of all kinds aim to encapsulate good practice in an easy-to-use format. They are specifically formulated to support interface designers in the complex and context-dependent task of interaction design (Abascal \& Colette, 2005; Cockton, 2004; Vogt, 1999). They seem to be particularly valuable when designing for special and unfamiliar contexts. Without guidance, the designer runs the risk of designing products for his or her own use (Cooper, 2004). Mere availability of published guidelines is clearly insufficient in terms of improving design. For a guideline to deliver value, it must be possible for designers to be able to: (1) identify the best set of guidelines; and (2) deploy them as the guideline developers anticipated. Both of these, it turns out, are problematical.

Renaud, K. and van Biljon, J. (2017). Demarcating mobile phone interface design guidelines to expedite selection . South African Computer Journal 29(3), 127-144. https://doi.org/10.18489/sacj.v29i3.438

Copyright (C) the author(s); published under a Creative Commons NonCommercial 4.0 License (CC BY-NC 4.0).

$S A C J$ is a publication of the South African Institute of Computer Scientists and Information Technologists. ISSN 1015-7999 (print) ISSN 2313-7835 (online). 
In terms of choice, users might well be unaware of the finer nuances of published guidelines. Their dimensions, such as purpose, focus and context, are seldom explicitly stated. It is thus entirely possible that designers, especially inexperienced ones, will inadvertently select and deploy guidelines that are not fit for purpose. For example, abstract heuristic guidelines, meant to guide interface evaluation, could erroneously be deployed to inform interface design. In this case specificity is essential and heuristics are too abstract. The resulting poor design can be attributed to guideline purpose mismatch (Bodart \& Vanderdonckt, 1993; Van Biljon \& Renaud, 2016). It is not necessarily the guideline itself that is flawed, but rather that there has been a mismatch in terms of purpose, context or any of the other dimensions that differentiate guidelines. The fact that such critical selection dimensions are not clearly stated, and might thus not be fully appreciated, could make guideline usage unlikely.

Even if the most appropriate set of guidelines is chosen, designers and developers seem to need extra support in deploying them (Zaphiris, Kurniawan, \& Ghiawadwala, 2007). There are particular difficulties related to developing and using guidelines proposed by third parties (Vogt, 1999). These difficulties are exacerbated by the dynamic and fluid nature of guidelines (Haines \& Feder, 1992), which change frequently in order to adapt to technological changes that affect mobile user interfaces.

The selection and deployment difficulties might well have a common cause: a failure to appreciate the multi-dimensional nature of the guidelines, and a consequent difficulty in choosing the right set of guidelines.

In the same way as there is no universally "good" feature in user interface design (Cockton, 2004), we argue that there is no universal guideline either. Those who labour under a "universal guideline" misapprehension cannot fully benefit from published guidelines, because they probably fail to appreciate their nuances and the complexities of the contexts within which they should or should not be followed. Guidelines are context-sensitive, nuanced and tailored to the specific needs of the context of use. Consequently, we argue that multiple descriptive dimensions of guidelines ought to be made explicit and salient when guidelines are published.

In this paper, an extension of our SAICSIT 2016 paper (Van Biljon \& Renaud, 2016), we report on a set of descriptive dimensions of guidelines that will expedite guideline choice and deployment. Our purpose was to encourage guideline creators explicitly to include guideline dimensions to support informed choice. If the critical dimensions for selecting an appropriate guideline are explicit, it ought to be easier for designers and developers to match a set of guidelines to their intended purpose and context of use.

Our research philosophy was pragmatist because this allowed us the flexibility to select the most appropriate method for each of the research phases. We carried out two investigations, taking snapshots of the field by carrying out first a scoping review and secondly a mapping systematic literature review.

The scoping review served to reveal an unstructured field with one-dimensional reporting of the guidelines, and confirmed a field of relative immaturity. This confirmed a clear need for a comprehensive set of guideline descriptions. During the subsequent mapping review we extracted a standard set of descriptive guideline dimensions to expedite guideline selection.

We conclude by detailing these descriptive guideline dimensions, which can be used to provide a 
structure and foundation for future guideline reporting. We thereby contribute towards maturation of the mobile interface design guideline research field.

\section{GUIDELINES AND RESEARCH DESIGN}

Many guidelines exist, covering a variety of design fields. We chose to focus on mobile phone design guidelines for three reasons.

The first is that mobile phones are ubiquitous, used by all cultures and languages across the globe. This makes mobile phone design especially topical.

The second reason is that, although phone interfaces have improved by leaps and bounds since the 1990s, they still sometimes fail to meet the needs of particular special-needs groups (Zaphiris et al., 2007). The speed of technological change, as well as physical and cognitive limitations, combine to marginalise some users. Guidelines do indeed exist to inform designers seeking guidance for these contexts: for instance, guidelines informing designing for senior citizens (Van Dyk, Gelderblom, Renaud, \& Van Biljon, 2013; Van Dyk, Renaud, \& Van Biljon, 2013). Despite these and other publications providing guidance for this particular context, there is little evidence that they have any impact on current mobile phone design.

The third reason was that our previous investigations had revealed that the mobile interface design guidelines mentioned in the literature were often related to the support of initial design rather than deployment activities (Van Biljon \& Renaud, 2016). These nuances are not necessarily obvious to the novice reader.

\subsection{Existing taxonomies}

Our aim in carrying out this investigation was to arrive at a set of dimensions to inform the selection of "fit for purpose" guidelines (Figure 1). We were unable to find a mobile phone guideline or interaction design guideline taxonomy. This suggests that any classifications that have been applied have not been made explicit, nor are they in general use. The taxonomies we did find were on a more detailed cognitive level, e.g. a taxonomy of user interface dimensions for consumer electronic devices (Kang \& Kim, 2007), a taxonomy of user interface terminologies (Chignell, 1990) or a taxonomy for mobile computation of mobile and context-aware devices (Rodden, Cheverst, Davies, \& Dix, 1998). Preece and Rombach (1994) published a taxonomy for combining software engineering and human-computer interaction measurements. This provided us with a point of departure in identifying categories towards arriving at a taxonomy for mobile phone design guidelines.

\subsection{Scoping review process}

We commenced by carrying out a scoping systematic literature review. A systematic literature review entails gathering research, removing duplicates, redundant and irrelevant papers and then summarising the best of what remains (Grant \& Booth, 2009). The systematic quantitative literature review method generally has three functions (Pickering, Grignon, Steven, Guitart, \& Byrne, 2015): 


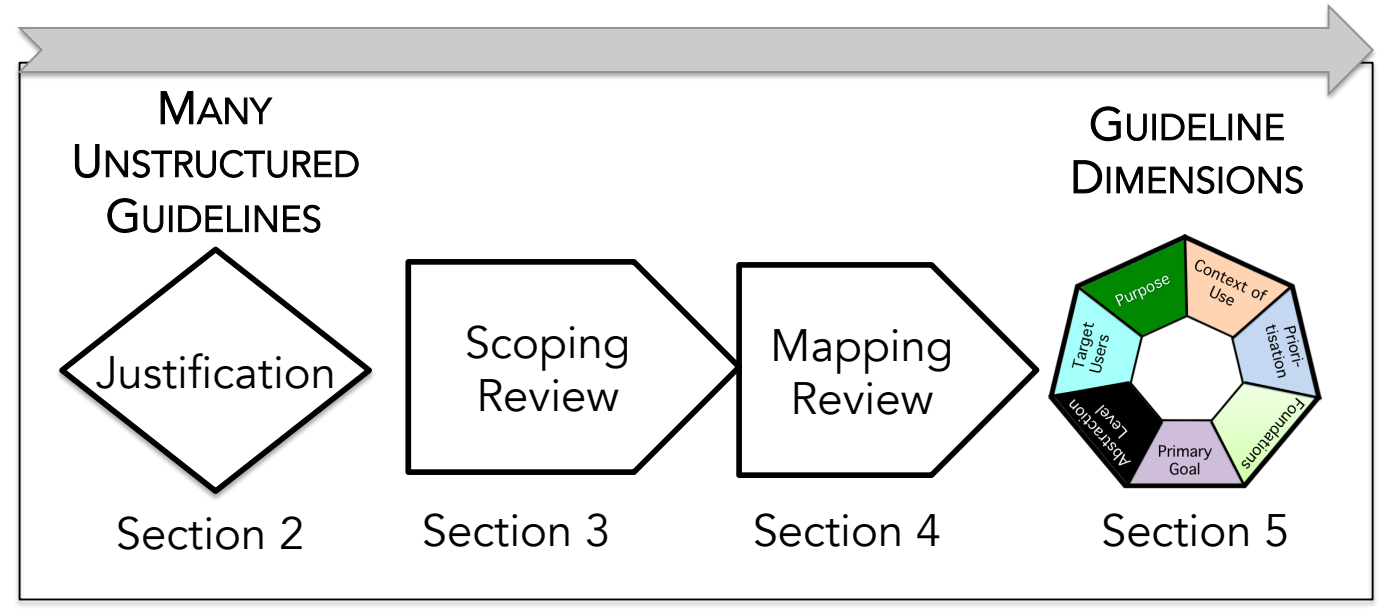

Figure 1: Research process diagram

1. identifying, summarising and critiquing current theory and methods,

2. identifying ontological, epistemological and methodological problems and gaps, and

3. providing evidence for decision-makers when identifying and supporting priority issues.

Grant and Booth (2009) distinguished fourteen types of interviews according to their search, appraisal, synthesis and analysis descriptions. Using their typology, we conducted a scoping review. Our scoping review investigated the range of publications in the mobile design guideline area in order to assess the maturity of the research field. Maturity is evidenced by a field starting to make an impact on industry, and building on other research in an area (Bødker, 2006, 2015; Harrison, Tatar, \& Sengers, 2007). The literature search was performed during November 2016.

Our analysis revealed an unstructured field, characterised by a large range of guidelines, but without a clear way for designers to choose between them.

We proceeded with a subsequent review to derive a set of descriptive dimensions that could be used to differentiate guidelines from each other, thereby expediting choice (Iyengar \& Lepper, 2000) and minimising guideline purpose mismatch.

\subsection{Mapping review process}

The second phase necessitated a systematic literature search and review. We wanted to demarcate the guideline research field in terms of current publications, the purpose, research methods and results. We carried out a mapping study, which is intended to review a topic by identifying, analysing and contemplating the extant studies in the area with a view to deriving a comprehensive set of guideline dimensions. The analysis was performed in July 2017 and resulted in a set of descriptive guideline dimensions. 
We conclude this paper by proposing the set of dimensions we derived. These can be used to describe and contextualise published guidelines. This, we believe, will bring structure to the field, encourage growth and engender maturity.

\section{SCOPING REVIEW}

Research fields progress and mature over time in terms of the kinds of research that is published (Cheon, Sabherwal, \& Groven, 1993). Some mature to merit independent status as a legitimate offshoot of the 'parent' field, while others shine briefly but then wither and stagnate. It could be argued that the changing nature of the published papers can be used as an indicator of how a field is maturing (Bødker, 2015). When a new human-related research field emerges, the initial wave of papers generally present individual, small-scale studies (Bødker, 2006). Researchers break ground and report empirical results in order to lay down founding principles. After a number of years, the research starts to mature and the second wave emerges, with reports on the use of the initial results in larger-scale studies, often in organisations: the focus on the individual no longer dominates. Social aspects start to merit inclusion, and initial results are used in further studies - building on the foundations laid during the initial period. Finally, the field displays adulthood when a third wave appears: meaning-making papers start appearing. Experienced researchers produce papers questioning unwritten assumptions and making recommendations about the way forward. The field starts to make an impact on people's lives. Harrison et al. (2007) also propose a maturation progression, with the final one also reflecting a meaning-making phase.

Renaud and Flowerday (2017) took snapshots of the CHI conference, the top HCI conference, for three years - 2004, 2010, and 2016 - to determine whether the published research reflected these waves. They classified the papers as belonging to one of the three waves, depending on whether the paper focused on the individual (1st wave), collaboration (2nd wave) or meaning making (3rd wave). Their analysis revealed an emergence of second and third wave papers over the last 12 years, confirming Bødker's assertions (2015) about the fact that published papers constitute evidence of the maturity of the HCI research field.

We considered that it would be helpful to examine the mobile interface guideline literature in the same way. Since Bødker (2015) and Harrison et al. (2007) had given their insights into how HCI had matured, we could use their blueprint to see whether the same kinds of maturing processes were emerging in mobile design research. This would help us to gauge the development and maturity of the field and suggest directions for future endeavours.

We searched for all mobile phone design guideline-related publications since 2010 in Scopus, Web of Science and ACM using the search string 'mobile phone' and 'design' and 'guideline'. We then used Google Scholar to identify references that did not appear in these databases. Table 1 lists the databases we searched and the number of relevant papers we found, discarded and retained based on a review of the abstracts.

The databases do not all have the same search facilities and that complicated the comparison to a certain extent. For example, the ACM digital library returned a large number of papers (358) because it was not possible to group words into searchable phrases. Some referred to 'design guidelines' in the 
Table 1: Outcome of literature search

\begin{tabular}{|l|ccc|}
\hline Databases & Papers returned & Papers discarded & Papers analysed \\
\hline Scopus & 90 & 31 & 59 \\
Web of Science & 30 & 29 & 1 \\
ACM & 358 & 345 & 13 \\
Google Scholar & 143 & 57 & 86 \\
\hline Total & & & 159 \\
\hline
\end{tabular}

keywords while in the other papers the keywords 'design' and 'guidelines' appeared separately. This included many papers that were not about mobile interaction design, and these were thus discarded. The search string also returned patents and chapters. These, and duplicates, were removed, leaving 159 papers to analyse.

Because the focus was on guidelines, we classified the papers as follows:

First Wave. Derivation of a set of mobile design guidelines, either by reviewing literature, by developing an interface, or by using surveys.

Second Wave. The focus here was on the organisations making use of guidelines, or researchers implementing pre-existing published guidelines in order to validate them.

Third Wave. What characterises this wave is meaning making. These papers focus on user experience, in this case the experiences of designers using guidelines, or the experiences of mobile phone owners using their mobile devices during their everyday lives, their user experience (with a focus on the interface design). Here we also included studies that took a meta-view of a particular aspect, or studies that analysed a number of studies and extracted principles for design - a less applied approach than waves 1 and 2, with a focus on extracting lessons to be learned.

The authors of this paper independently mapped the remaining papers to the three waves and then met to agree on final classifications. Seventy-four papers were eliminated at this stage because they did not implement or derive guidelines, leaving 85 to be classified.

Figure 2 showed that first wave papers dominate, more so than in the parent HCI field (see Renaud and Flowerday (Renaud \& Flowerday, 2017)). Only a handful of second and third wave papers have been published since 2010. What also became clear is that many researchers were conducting studies to generate guidelines, but only a handful implemented pre-existing mobile phone guidelines or created meaning in terms of how the guidelines were being used by the community, or considered how the interfaces were being used as artefacts by the developer and designer community. The consequence is that there are multiple proposed guidelines to choose from, but no easy way to inform those wishing to find the right set of guidelines to use. This suggests that meaning-making publications in the field are rare. 


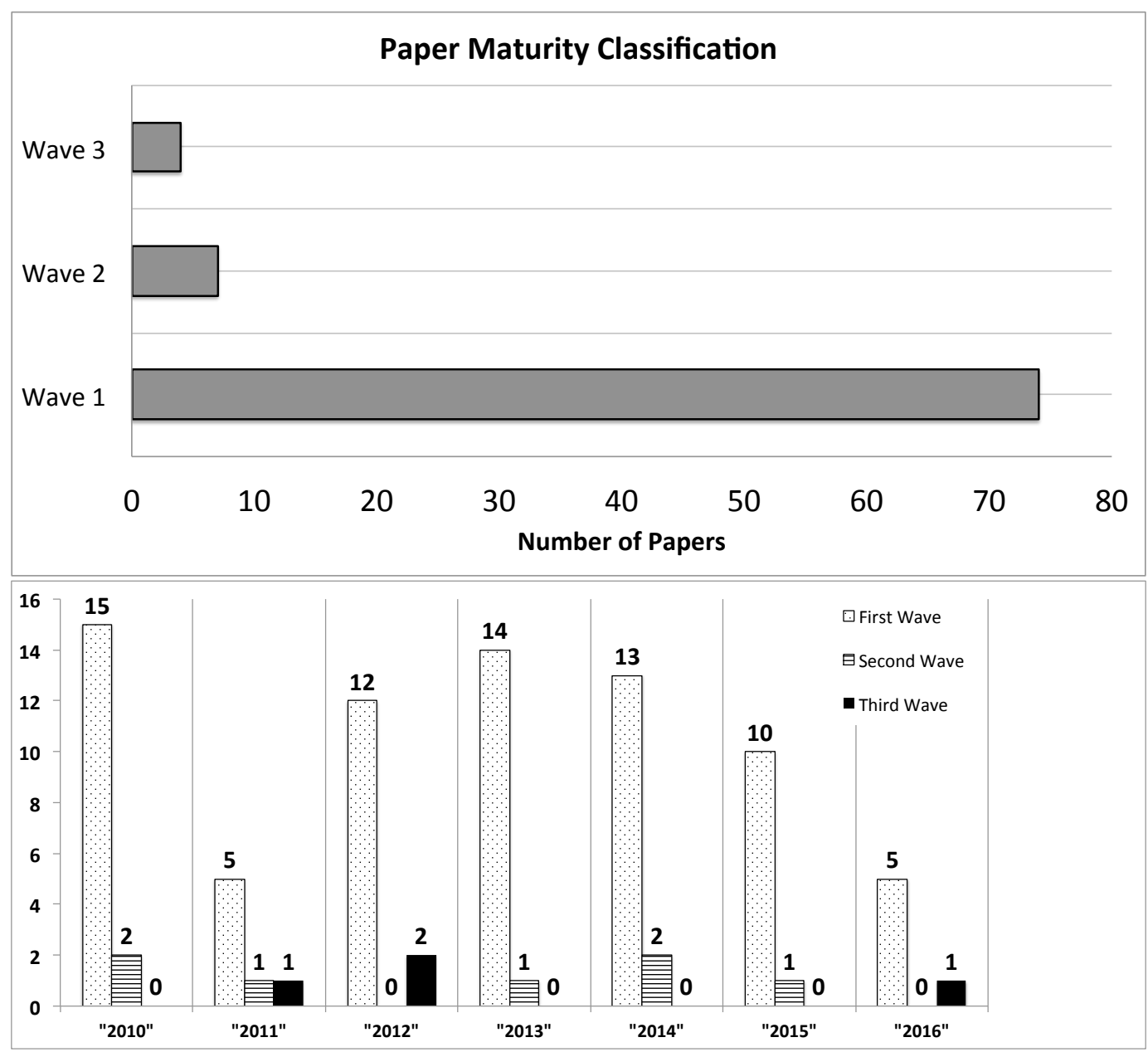

Figure 2: The numbers of mobile phone interface guideline papers classified into waves: (1: new guidelines; 2: validating/using guidelines; 3: user experience)

We also noticed, as we read and analysed the abstract, that the guidelines themselves, and their context of use, was seldom explicitly stated and often had to be derived from the surrounding text. It was difficult to find their descriptive dimensions in some cases.

To encourage maturity of the field as a whole, it would be helpful to have a descriptive structure, a set of dimensions, to encourage guideline deployment and incremental refinement and improvement of guidelines. The following section details the process we undertook to derive a descriptive set of dimensions. 


\section{MAPPING REVIEW}

Using the search string ("mobile phone" or "cell phone") and "interface design guidelines" we searched for all mobile phone interface design guideline related publications since 2007 in Scopus, Web of Science, DOAJ, ACM and Springer. We used Google Scholar to identify papers that did not appear in these databases. This search was narrower than the previous one, because here we were focusing primarily on interface design. Table 2 lists the databases we searched and the number of relevant papers we found, discarded and retained. We discarded papers that did not have anything to do with the topic in question. After the qualitative analysis we narrowed the analysis further by discarding papers that were not related specifically to guideline generation or at least derived, or made use of, a set of guidelines.

Table 2: Outcome of literature search

\begin{tabular}{|l|cccc|}
\hline Databases & Papers returned & Papers discarded & $\begin{array}{l}\text { Papers } \\
\text { analysed }\end{array}$ & $\begin{array}{l}\text { quantitatively } \\
\text { analysed }\end{array}$ \\
\hline Scopus & 2 & 1 & 1 & 0 \\
Web of Science & 1 & 0 & 1 & 2 \\
ACM & 17 & 1 & 16 & 2 \\
Springer & 28 & 2 & 26 & 10 \\
DOAJ & 3 & 0 & 3 & 0 \\
Google Scholar & 46 & 29 & 17 & 6 \\
\hline Total & & & 64 & 18 \\
\hline
\end{tabular}

\subsection{Quantitative analysis}

The full papers were read and analysed individually to extract descriptive details. Of the 65 papers, 49 were published in conferences, and 16 in peer-reviewed journals. The authors' countries are depicted in Figure 3.

Figure 4 depicts the stated research type, and the number of citations for each category. We used Google Scholar to determine the number of citations for each of the papers in our analysed corpus.

Table 3 provides the descriptive statistics for paper citations. Only one paper was highly cited (Tsai et al., 2007): a paper reporting on a mobile phone application for monitoring real time caloric balance. It received 224 citations. The authors did not derive or evaluate guidelines.

Table 3: Number of citations

\begin{tabular}{|l|cccc|}
\hline & Min & Max & Mode & Median \\
\hline All papers & 0 & 224 & 0 & 6 \\
& 11 papers & 1 paper & & 3 papers \\
\hline $\begin{array}{l}\text { Papers that derive and evaluate the } \\
\text { guidelines }\end{array}$ & 0 & 36 & 0 & 15 \\
& 2 papers & 1 paper & & \\
\hline
\end{tabular}




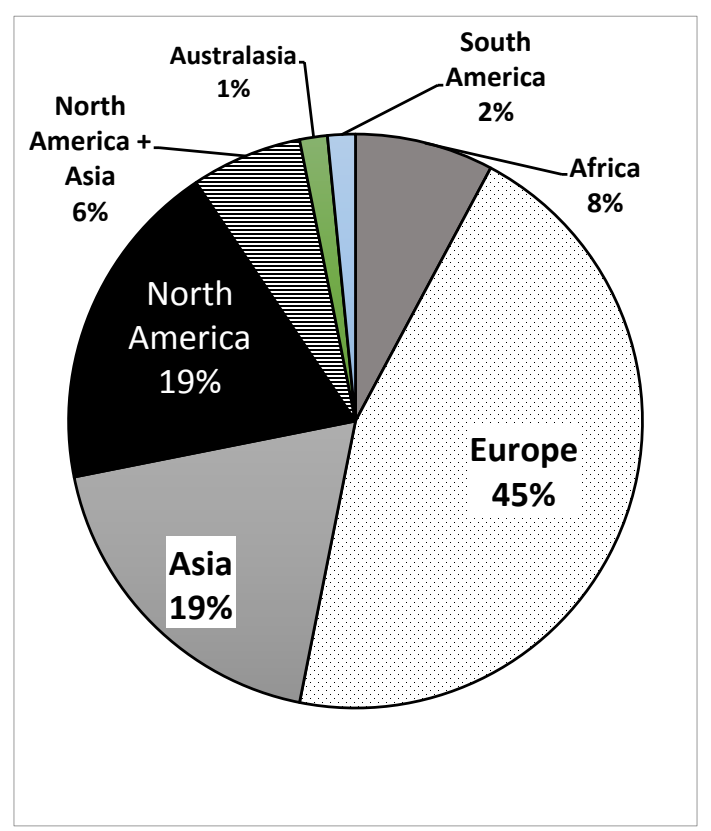

Figure 3: Authors' countries

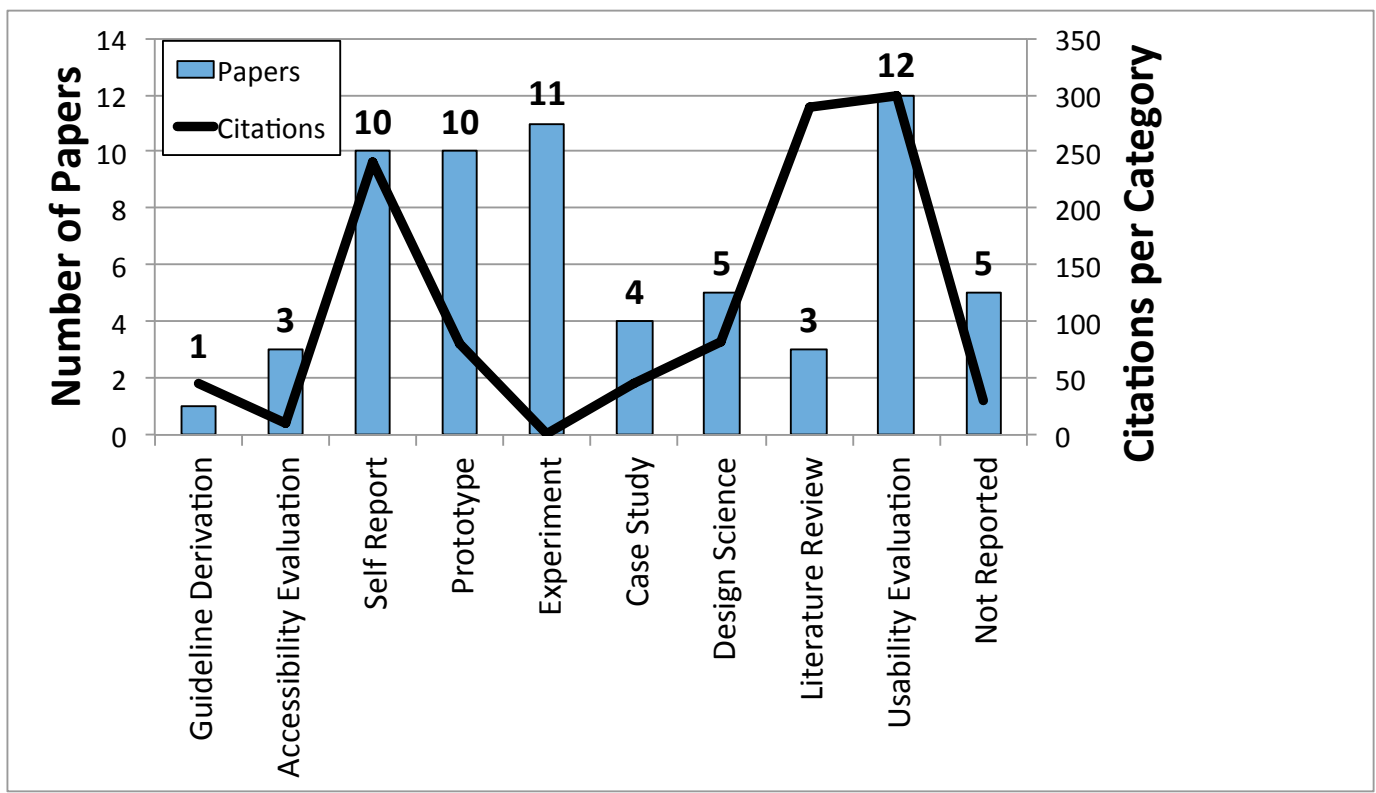

Figure 4: Research types and citation numbers 
Figure 5 shows the number of citations per paper, and clearly demonstrates the immaturity of the field. With only 3 papers receiving more than 50 citations, it does not seem as if researchers were building on each other's work during the decade of focus (2007-2017). This raises questions about the usefulness of guidelines beyond mere publication of executed research.

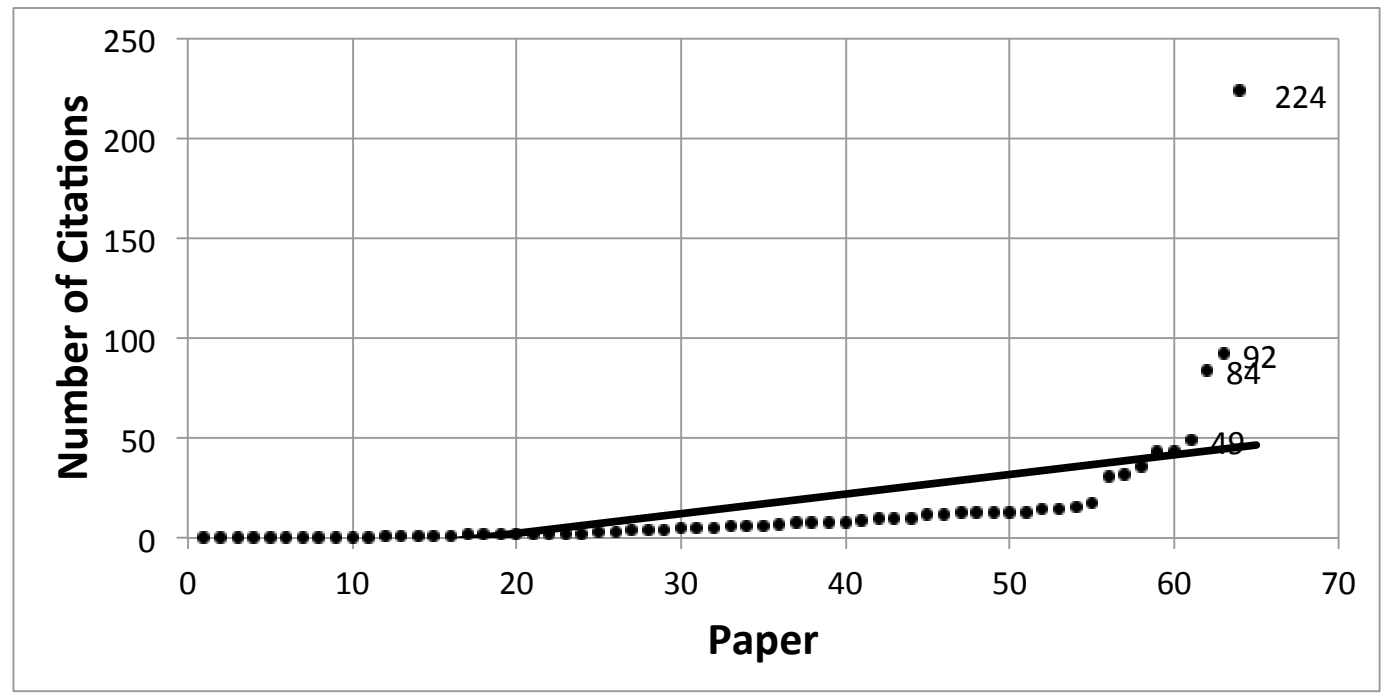

Figure 5: Number of citations per paper

Figure 6 suggests that, after an initial flurry of interest in guideline papers, the field has been characterised by a sustained interest from researchers who do not seem to be refining or improving other published guidelines but rather developing new ones. This is a significant observation since research is supposed to extend knowledge ("If I have seen further it is by standing on ye sholders of Giants". (Newton, 1675)). It is likely that many factors contribute to the relative stagnation of the field. The fact that 49 of the 65 papers were published in conferences may provide some explanation because conference publications are sometimes less accessible and noticeable than journal papers. It could also be that researchers found it difficult to find or discriminate between the guidelines that did exist and decided to generate new ones that suited their context. The second explanation could be addressed by providing better descriptions of the guidelines. We thus carried out a qualitative analysis to derive a set of descriptive dimensions.

\subsection{Qualitative analysis}

We selected only those papers that derived or evaluated guidelines. These were then analysed to extract the purpose of the guideline (different from the purpose of the paper), the abstraction level (abstract of specific guidance), the target group and whether there was any attempt at prioritisation.

A total of 18 papers were analysed. Guidelines were derived in 13 of the papers, and evaluated in 13 of the papers. Seven papers derived and evaluated guidelines. Two papers reported industry involvement. 


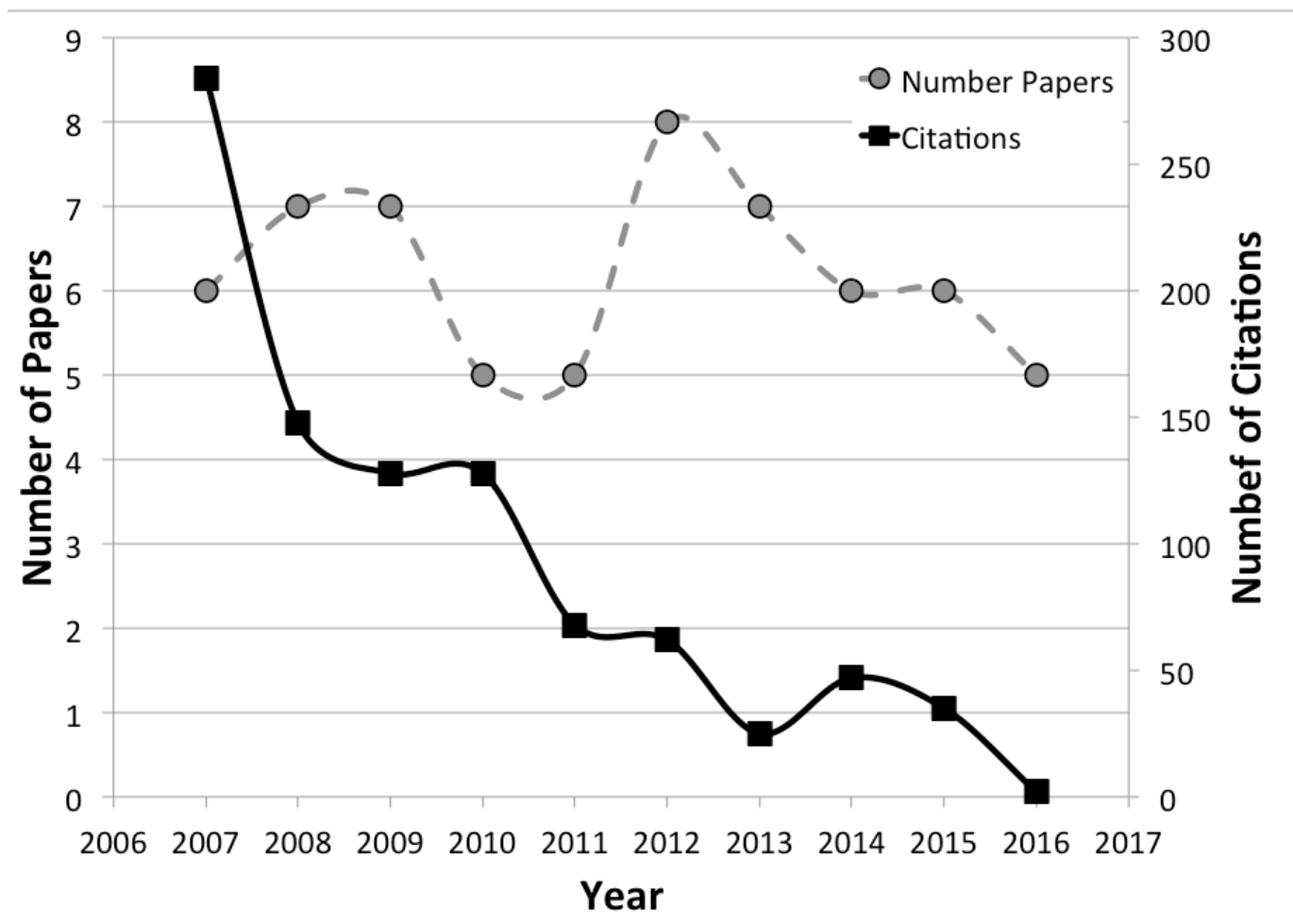

Figure 6: Number of citations per publication year

The dimensions identified in Preece and Rombach's taxonomy (1994) were used to guide the thematic analysis. Information related to that dimension (label) was extracted and new dimensions that emerged augmented the list. Preece and Rombach identified goal, plan, methods and techniques as the primary dimensions, with secondary dimensions for each of those.

Purpose. Vanderdonckt (1999) mentioned the following five phases for using guidelines: (1) the specification phase where a set of guidelines is delimited as requirements for the future UI; (2) the design phase; (3) the prototyping phase, (4) the evaluation phase; and (5) the documentation and certification phase where guidelines are applied for documenting an interactive application for communication, reuse, maintenance or commercial promotion purposes. These development phases relate to the overall purpose of guidelines as delineated by Preece and Rombach (1994). When reading the papers to extract the purpose of the guidelines, the emergent categories were: (1) requirement gathering; (2) design, (3) evaluation and (4) organisation. We categorised each of the papers to uncover other purposes. The results were as follows:

- Requirements gathering (Koc, Cikrikcili, Yucel, Cheng, \& Salman, 2012; Mi, Cavuoto, Benson, Smith-Jackson, \& Nussbaum, 2014; Schneidermeier, Burghardt, \& Wolff, 2013);

- Design: (Abdulrazak, Malik, Arab, \& Reid, 2013; Ahmad, Komninos, \& Baillie, 2008; Ammar, 
Trabelsi, \& Adel, 2015; Bezold \& Minker, 2011; Gkatzidou et al., 2015; Jeong, Proctor, \& Salvendy, 2009; Lokman, Noor, \& Nagamachi, 2009; Massimi, Baecker, \& Wu, 2007; Tarasewich, Gong, Nah, \& DeWester, 2008; Yeratziotis \& Van Greunen, 2013);

- Evaluation (Chignell, 1990; Fakun, 2008; Van Biljon \& Renaud, 2016; Grammenos, Kartakis, Adami, \& Stephanidis, 2008; Masip, Oliva, \& Granollers, 2011); and

- Organisation (Nicastro et al., n.d.).

All papers could be classified into one of these categories. Given the initial search string, we expected all the papers to focus on design, so finding papers focused on requirements gathering, organisation and evaluation (without any design content) was surprising. This confirmed our initial impression that guidelines are inadequately labelled, which makes them hard to differentiate and deploy.

Specificity. Guidelines are presented at different levels of abstraction. For example, a a design guideline is more specific than a design principle ${ }^{1}$. The terminology for a rising level of specification is not consistently followed in the guideline literature (Winters \& Toyama, 2009). For the purpose of this analysis we used only two levels, namely abstract or specific. Ten papers were classified as abstract and eight as specific. That is admittedly subjective but the important issue is that, whatever the split, the papers were at different levels of abstraction. This impacts their utility and fitness for purpose.

Target Users. It is important to identify the target user base: those for whom the guidelines were developed. This was seldom explicitly stated, but we were able to derive the intended users from the guideline purpose. The guidelines aimed at requirements gathering and design were intended for use by developers (although users were obviously involved in requirements gathering). The evaluation guidelines were for developers and usability evaluators involved in testing the user interfaces.

Context of Use. In terms of guidelines, this reflects the intended end-users of the product to be specified, developed or improved by the guidelines. The contexts of the analysed papers include: senior citizens (3); differently-abled (3); students (6); and other target groups, such as managers in organisations.

Prioritisation. In Software Engineering, it is common to prioritise features using a technique such as MoSCoW (must have, should have, could have, won't have). It is telling that only two of the guidelines attempted to prioritise guidelines. Other papers did not mention prioritisation so they appear to have ranked all guidelines equally. This provides further evidence to confirm the relative immaturity of the field since there is no evidence of appreciation of the reality of real-world deployment, where it is often impossible to implement all possible guidelines. In these cases the designer has to satisfice. Some guidance, to inform guideline selection, would be helpful.

\footnotetext{
${ }^{1}$ http://alandix.com/blog/2016/03/31/principles-vs-guidelines/

${ }^{2}$ https://en.wikipedia.org/wiki/MoSCoW_method
} 
Foundations. An issue that emerged from the analysis is the lack of alignment between academia and industry. We noticed that there was little reference to industry guidelines despite the fact that several sets exist. One example is Google's Material Design Guidelines, designed to support the latest version of Android ${ }^{3}$. One explanation for this omission could be that the commercial guidelines are too generic for specific demographics. A guideline stating that all icons should be accompanied by text is problematic to implement on a mobile phone interface where there are two opposing constraints. For example, space might be limited but there is also a need for the font to be big enough to be read by the visually challenged. Moreover, if target users are illiterate such a guideline is counterproductive. Finally, in multilingual countries you would also have to provide a setup to allow the new owner to choose a language, and this adds an extra level of complexity to its design, implementation and use.

\subsection{Final dimensions}

This investigation led to the following recommendations in terms of the following descriptive guideline dimensions (Figure 7).

1. Purpose. The following, non-exhaustive sub-dimensions are suggested as a point of departure: (a) requirements gathering; (b) design; (c) evaluation; and (d) organisation.

2. Level of abstraction. The coarsest possible distinction is abstract or specific but finer distinctions might emerge as these dimensions are augmented in the future. Abstract would be: 'choose colours with colour-blind people in mind'. Specific would be: 'never superimpose red on green, and vice versa'.

3. Target Users. For whom were the guidelines developed? Usability analysts, interaction (including interface) designers, testers or developers.

4. Context of Use. Who are the intended end-users of the product? Eg. for special needs groups, a particular demographic, or for specific hardware such as a specific phone or robot?

5. Prioritisation. Are guidelines prioritised? Do the guidelines help the designer to prioritise?

6. Primary goal. This could be one of the following: (a) usability maximisation; (b) accessibility improvement; (c) cost reduction; (d) user experience maximisation; (e) requirements gathering optimisation; (f) usability evaluation; ( $\mathrm{g}$ ) accessibility evaluation; and (h) other. This list is bound to be extended as the field matures.

7. Foundations. Which sets of guidelines are used and/or refined by the research, either industry based or those from previous publications? This would help to indicate the maturity of a specific set of guidelines.

\footnotetext{
${ }^{3}$ See https://design.google.com/resources/ and the Material Design Guidelines to inform icon design https: //material.google.com/style/icons.html.
} 
If researchers explicitly include guideline dimensions in their reports, it will be much easier for designers and developers to find the best possible set of guidelines for their particular context.

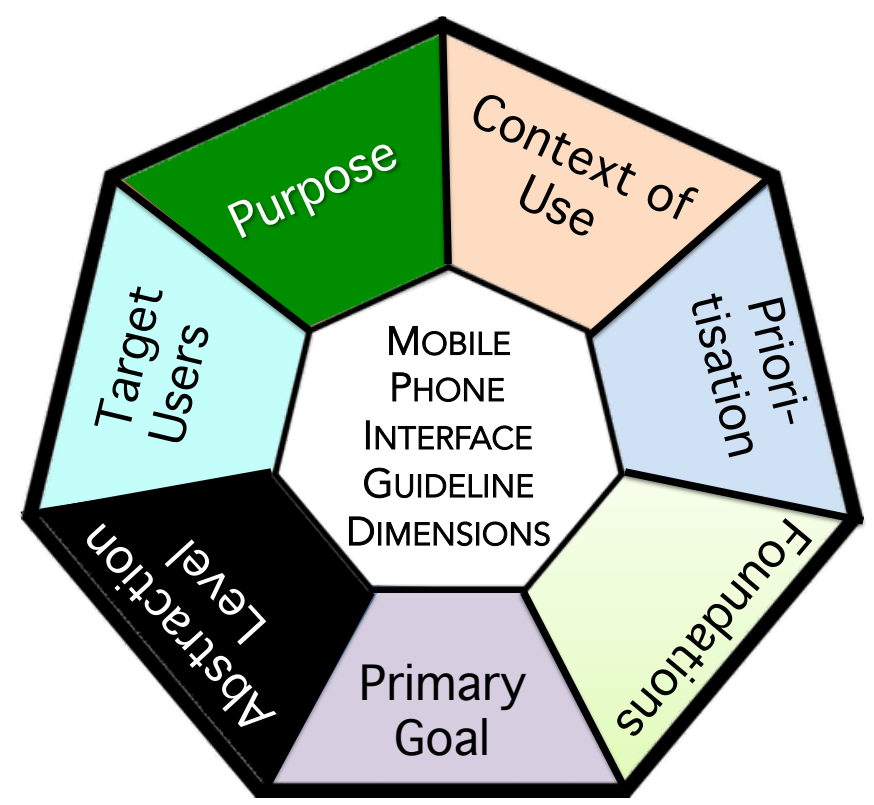

Figure 7: Mobile phone interface design guideline dimensions

\subsection{Limitations}

During our scoping review we classified the papers into one of three waves. The papers were classified independently by the two researchers who then met to agree on final classifications. This served to address individual subjectivity but it is entirely possible that the papers would have been classified differently by a different set of researchers. However, this research sought to identify trends and we believe that we were able to do so. The mapping review offered fewer opportunities for subjectivity. During this phase, too, two researchers independently extracted dimensions and then met to agree on the final dimensions.

\section{CONCLUSION}

The research field we have examined is currently characterised by a proliferation of guidelines with no over-arching structure to support informed selection of the most appropriate set of guidelines, nor any indication of their usefulness in an intended context of use.

The scoping review demonstrated that despite many publications in academic conferences and journals, the field was immature in terms of publications building on extant knowledge, as measured by citations. There could be many reasons for this non-uptake, but the one we focused on was our 
observation that the guidelines were reported in a one-dimensional fashion. They were seldom contextualised and their nuances and fitness for particular purposes and uses were seldom explicitly reported.

We carried out a systematic literature review of all the research related to the narrowed down field of mobile phone interface guideline research over the last decade. We derived a set of descriptive dimensions which could be used to impose a structure onto the field of guideline research, as depicted in Figure 7.

Considering that none of the published guidelines made all these dimensions explicit, the value of this paper is to create awareness of what to look for when selecting a mobile phone design guideline. We also propose that future guideline papers of all kinds consider including descriptive dimensions to improve discoverability and utility.

\section{ACKNOWLEDGEMENTS}

This work is based on the research supported by the South African Research Chairs Initiative of the Department of Science and Technology and National Research Foundation of South Africa (Grant No. 98564). We acknowledge Louisa Rabalao and Thabo Lehong for their assistance in analysing the data.

\section{References}

Abascal, J. \& Colette, N. (2005). Moving towards inclusive design guidelines for socially and ethically aware HCI. Interacting with Computers, 17(5), 484-505. https://doi.org/10.1016/j.intcom. 2005.03.002

Abdulrazak, B., Malik, Y., Arab, F., \& Reid, S. (2013). PhonAge: Adapted smartphone for aging population. In International Conference on Smart Homes and Health Telematics (pp. 27-35). https://doi.org/10.1007/978-3-642-39470-6_4

Ahmad, D., Komninos, A., \& Baillie, L. (2008). Future mobile health systems: Designing personal mobile applications to assist self diagnosis. In Proceedings of the 22nd British HCI Group Annual Conference on People and Computers: Culture, Creativity, Interaction: Volume 2 (pp. 39-42). British Computer Society.

Ammar, L. B., Trabelsi, A., \& Adel, M. (2015). Incorporating usability requirements into model transformation technologies. Requirements Engineering, 20(4), 465-479. https://doi.org/10. 1007/s00766-014-0213-z

Bezold, M. \& Minker, W. (2011). Adaptation patterns for interactive systems. In Adaptive multimodal interactive systems (pp. 67-80). https://doi.org/10.1007/978-1-4419-9710-4

Bodart, F. \& Vanderdonckt, J. [J.M.]. (1993). Expressing guidelines into an ergonomical styleguide for highly interactive applications. In INTERACT '93 and CHI '93 conference companion on Human factors in computing systems - CHI '93 (pp. 35-36). ACM. https://doi.org/10.1145/259964. 260036 
Bødker, S. (2006). When second wave HCI meets third wave challenges. In Proceedings of the 4th Nordic conference on Human-computer interaction: changing roles (pp. 1-8). ACM. https: //doi.org/10.1145/1182475.1182476

Bødker, S. (2015). Third-Wave HCI, 10 years later. Interactions, 22(5), 24-31. https://doi.org/10. $1145 / 2804405$

Cheon, M., Sabherwal, R., \& Groven, V. (1993). The evolution of empirical research in IS: A study in IS maturity. Information and Management, 24(3), 107-119. https://doi.org/10.1016/03787206(93)90060-7

Chignell, M. (1990). A taxonomy of user interface terminology. SIGCHI Bulletin, 21(4), 27-34. https://doi.org/10.1145/379106.379114

Cockton, G. (2004). From quality in use to value in the world. In CHI'04 extended abstracts on Human factors in computing systems (pp. 1287-1290). https://doi.org/10.1145/985921.986045

Cooper, A. (2004). The inmates are running the asylum. Sams Publishing. https://doi.org/10.1007/ 978-3-322-99786-9

Fakun, D. (2008). A review of issues surrounding the evaluation of industrial hypermedia applications. International Journal of Advanced Manufacturing Technology, 39(7-8), 828-837. https://doi. org/10.1007/s00170-007-1265-8

Gkatzidou, V., Hone, K., Sutcliffe, L., Gibbs, J., Sadiq, S., Szczepura, A., ... Estcourt, C. (2015). User interface design for mobile-based sexual health interventions for young people: Design recommendations from a qualitative study on an online chlamydia clinical care pathway. BMC Medical Informatics and Decision Making, 15(72), 1-13. https://doi.org/10.1186/s12911015-0197-8

Grammenos, D., Kartakis, S., Adami, I., \& Stephanidis, C. (2008). CAMILE: Controlling AmI lights easily. In Proceedings of the 1st ACM international conference on Pervasive Technologies Related to Assistive Environments - PETRA '08 (pp. 1-8). ACM. https://doi.org/10.1145/1389586. 1389628

Grant, M. \& Booth, A. (2009). A typology of reviews: An analysis of 14 review types and associated methodologies. Health Information and Libraries Journal, 26(2), 91-108. https://doi.org/10. 1111/j.1471-1842.2009.00848.x

Haines, A. \& Feder, G. (1992). Guidance on guidelines. BMJ: British Medical Journal, 305, 785-786. https://doi.org/10.1136/bmj.305.6857.785

Harrison, S., Tatar, D., \& Sengers, P. (2007). The three paradigms of HCI. In Alt. Chi. Session at the SIGCHI Conference on Human Factors in Computing Systems (pp. 1-18).

Iyengar, S. \& Lepper, M. (2000). When choice is demotivating: Can one desire too much of a good thing? Journal of Personality and Social Psychology, 79(6), 995-1006. https://doi.org/10. 1037/0022-3514.79.6.995

Jeong, K., Proctor, R., \& Salvendy, G. (2009). A survey of smart home interface preferences for US and Korean users. In Proceedings of the Human Factors and Ergonomics Society 53rd Annual Meeting (pp. 541-545). https://doi.org/10.1177/154193120905300812

Kang, S. \& Kim, W. (2007). Minimalist and intuitive user interface design guidelines for consumer electronics devices. Journal of Object Technology, 6(3), 39-52. 
Koc, Y., Cikrikcili, O., Yucel, A., Cheng, H.-I., \& Salman, Y. (2012). A mobile course management system: Case study. In Proceedings 6th International Conference, ICHIT 2012, August 2012 (pp. 377-385). https://doi.org/10.1007/978-3-642-32692-9_47

Lokman, A., Noor, N., \& Nagamachi, M. (2009). Expert Kansei web: A tool to design Kansei website. In International Conference on Enterprise Information Systems (LNBIP, volume 24) (pp. 894-409). https://doi.org/10.1007/978-3-642-01347-8_74

Masip, L., Oliva, M., \& Granollers, T. (2011). Classification of interactive system components enables planning heuristic evaluation easier. In International Conference of Design, User Experience, and Usability (pp. 478-486). https://doi.org/10.1007/978-3-642-21708-1_54

Massimi, M., Baecker, R., \& Wu, M. (2007). Using participatory activities with seniors to critique, build, and evaluate mobile phones. In ASSETS'07 (pp. 155-162). https://doi.org/10.1145/ 1296843.1296871

Mi, N., Cavuoto, L., Benson, K., Smith-Jackson, T., \& Nussbaum, M. (2014). A heuristic checklist for an accessible smartphone interface design. Universal Access in the Information Society, 13(4), 351-365. https://doi.org/10.1007/s10209-013-0321-4

Newton, I. (1675). Letter from Sir Isaac Newton to Robert Hooke. Last accessed 03 Dec 2017. Retrieved from http://digitallibrary.hsp.org/index.php/Detail/Object/Show/object_id/ 9285

Nicastro, F., Pereira, R., Alberton, B., Morellato, L. P. C., Baranauskas, C., \& Torres, R. d. S. (n.d.). Guidelines for evaluating mobile applications: A semiotic-informed approach. In H. S., M. L., T. E., C. O., \& C. J. (Eds.), Enterprise information systems. lecture notes in business information processing, vol 241. https://doi.org/10.1007/978-3-319-29133-8

Pickering, C., Grignon, J., Steven, R., Guitart, D., \& Byrne, J. (2015). Publishing not perishing: How research students transition from novice to knowledgeable using systematic quantitative literature reviews. Studies in Higher Education, 40(10), 1756-1769. https:// doi.org/10. 1080/03075079.2014.914907

Preece, J. \& Rombach, H. (1994). A taxonomy for combining Software Engineering and HumanComputer Interaction measurement approaches: Towards a common framework. International Journal of Human-Computer Studies, 41 (4), 553-583. https://doi.org/10.1006/ijhc.1994. 1073

Renaud, K. \& Flowerday, S. (2017). Contemplating human-centred security and privacy research and suggesting future directions. Journal of Security and Applications, 34(1), 76-81. https: //doi.org/10.1016/j.jisa.2017.05.006

Rodden, T., Cheverst, K., Davies, K., \& Dix, A. (1998). Exploiting context in HCI design for mobile systems. In Workshop on Human Computer Interaction with Mobile Devices (pp. i21-22). Retrieved from http://citeseer.ist.psu.edu/viewdoc/summary?doi=10.1.1.57.1279

Schneidermeier, T., Burghardt, M., \& Wolff, C. (2013). Design guidelines for coffee vending machines. In A. Marcus (Ed.), Design, user experience, and usability. web, mobile, and product design. duxu 2013. lecture notes in computer science, vol 8015 (pp. 432-440). Springer. https://doi.org/10. 1007/978-3-642-39253-5_48 
Tarasewich, P., Gong, J., Nah, F.-H., \& DeWester, D. (2008). Mobile interaction design: Integrating individual and organizational perspectives. Information Knowledge Systems Management, 7, 121-144.

Tsai, C., Lee, G., Raab, F., Norman, G., Sohn, T., Griswold, W., \& Patrick, K. (2007). Usability and feasibility of PmEB: A mobile phone application for monitoring real time caloric balance. Mobile Networks and Applications, 12(2-3), 173-184. https://doi.org/10.1007/s11036-0070014-4

Van Biljon, J. \& Renaud, K. (2016). Validating mobile phone design guidelines: Focusing on the elderly in a developing country. In Proceedings of the Annual Conference of the South African Institute of Computer Scientists and Information Technologists - SAICSIT '16 (pp. 1-10). https: //doi.org/10.1145/2987491.2987492

Van Dyk, T., Gelderblom, H., Renaud, K., \& Van Biljon, J. (2013). Mobile phones for the elderly: A design framework. Proceedings of the 7th International Development Informatics Conference.

Van Dyk, T., Renaud, K., \& Van Biljon, J. (2013). MOSES - Method for selecting senior mobile phones: Supporting design and choice for the elderly. In SAICSIT '12 (pp. 277-285). https: //doi.org/10.1145/2389836.2389869

Vanderdonckt, J. [J.]. (1999). Development milestones towards a tool for working with guidelines. Interacting with Computers, 12(2), 81-118. https://doi.org/10.1016/S0953-5438(99) 00019-3

Vogt, T. (1999). The use of guidelines for designing user interfaces in commercial software development. Last accessed 03 Dec 2017. Retrieved from http://citeseerx.ist.psu.edu/viewdoc/ download?doi=10.1.1.167.23\&rep=rep $1 \&$ type $=$ pdf

Winters, N. \& Toyama, K. (2009). Human-computer interaction for development: Mapping the terrain. Information Technologies and International Development, 5(4), 3-8.

Yeratziotis, G. \& Van Greunen, D. (2013). Making ICT accessible for the deaf. In IST-Africa 2013 Conference Proceedings (pp. 1-9).

Zaphiris, P., Kurniawan, S., \& Ghiawadwala, M. (2007). A systematic approach to the development of research-based web design guidelines for older people. Universal Access in the Information Society, 6(1), 59-75. https://doi.org/10.1007/s10209-006-0054-8 